\title{
In Situ Transmission Electron Microscopy of Electrochemical Nucleation and Growth of Copper on Gold
}

\author{
A. Radisic, ${ }^{*}$ P. C. Searson, ${ }^{*}$ and F. M. Ross** \\ * Department of Materials Science and Engineering, Johns Hopkins University, 3400 N. Charles St. \\ Baltimore, MD 21218, USA \\ ** IBM T. J. Watson Research Center, Yorktown Heights, NY 10598, USA
}

Electrodeposition is the current method used in forming copper interconnects in integrated circuits, primarily due to the ability of this technique to fill high aspect ratio features with complex geometries at high deposition rates, leading to high throughput and lower manufacturing costs. The growth of copper films on dissimilar substrates, such as diffusion barrier materials, occurs through Volmer-Weber (3D island) growth. As a result, high nucleus densities are essential in depositing thin films. For complex structures with small length scales, such as trenches and vias in integrated circuits, a detailed understanding of nucleation and growth, and the influence of parameters such as potential and solution chemistry on the deposition mechanism is critical in designing processes for obtaining the void-free features.

The mechanism of nucleation and growth in electrochemical deposition of thin films is usually determined from analysis of electrochemical experiments, such as current - time transients, in conjunction with ex situ imaging of the surface. However, such techniques do not reveal the rates of growth of individual islands or the spatial correlations between islands.

We have studied nucleation and growth processes during electrochemical deposition of copper on gold using in situ transmission electron microscopy [1]. This is a real-time, high resolution imaging technique that allows us to study the evolution of individual islands with time, as well as the spatial and temporal correlations between islands. Furthermore, this in situ technique allows us to compare electrochemical parameters commonly used to determine growth mechanisms, such as current - time transients, with real time images of island growth.

Figure 1 shows a series of TEM images recorded simultaneously with current-time transients during deposition of copper from $100 \mathrm{mM} \mathrm{CuSO}_{4}$ solution $(\mathrm{pH}=1)$ at $-0.07 \mathrm{~V}(\mathrm{vs} \mathrm{Cu}$ wire). The images show that copper deposition under these conditions occurs through Volmer-Weber island growth. The island density increases with time (not shown) and reaches a maximum of $1 \times 10^{9} \mathrm{~cm}^{-2}$ after $1 \mathrm{~s}$.

In this study we report on the nucleation and growth parameters for copper electrodeposition, obtained using in situ experimental techniques. We examine the relationship between potential, solution chemistry, and island density. We show that under appropriate experimental conditions, a high density of copper islands and a continuous thin copper film can be obtained at the surface.

References

[1] F. M. Ross, IBM Journal of Research, 44 (2000) 489. 


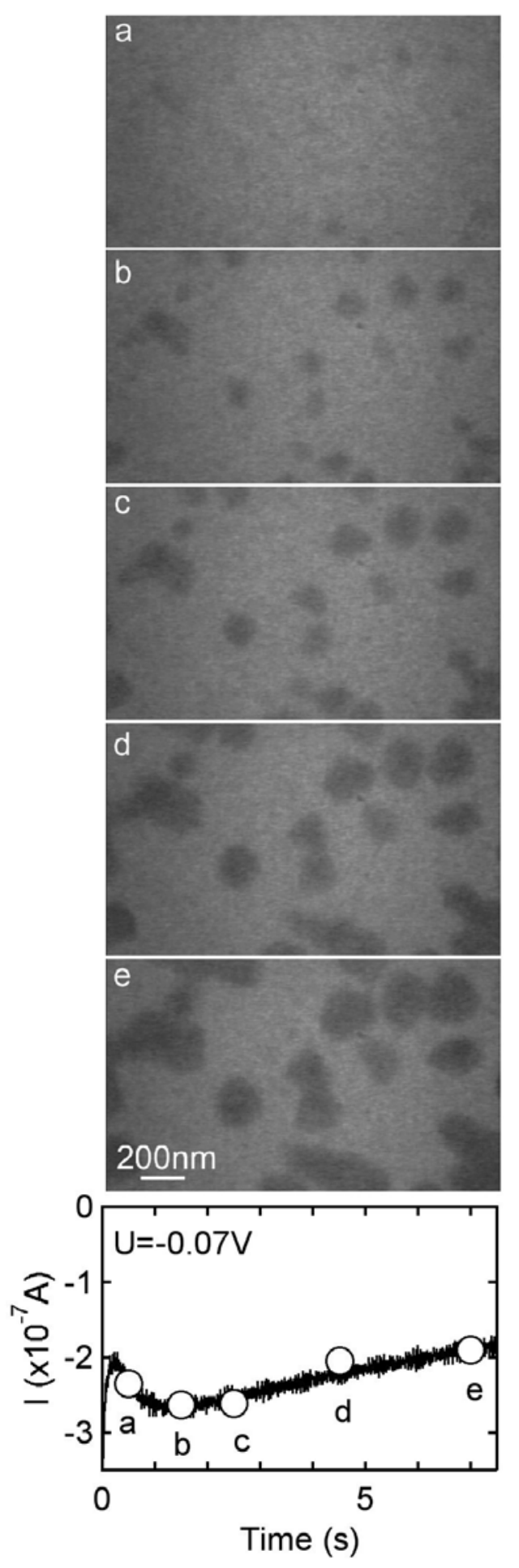

Figure 1. A sequence of images recorded simultaneously with deposition at $-0.07 \mathrm{~V}$. In order to record a current-time transient, the potential is first stepped from the open circuit potential $(\approx 0.04$ $\mathrm{V})$ to $0.15 \mathrm{~V}$ for 2 seconds to ensure that there is no copper on the gold surface. The potential was then stepped to a value of $-0.07 \mathrm{~V}$ where deposition occurs. 